\title{
Application of Homotopy to the Ageing Process of Human Body Within the Framework of Algebraic Topology
}

\author{
Lewis Brew ${ }^{1}$, William Obeng-Denteh ${ }^{2} \&$ David Delali Zigli $^{1}$ \\ ${ }^{1}$ Department of Mathematical Sciences, University of Mines and Technology, Tarkwa, Ghana \\ ${ }^{2}$ Department of Mathematics, Kwame Nkrumah University of Science and Technology, Kumasi, Ghana \\ Correspondence: Lewis Brew, Department of Mathematical Sciences, University of Mines and Technology, Tarkwa, \\ Ghana.
}

Received: May 30, 2019 Accepted: July 2, 2019 Online Published: July 11, 2019

doi:10.5539/jmr.v11n4p21

URL: https://doi.org/10.5539/jmr.v11n4p21

\begin{abstract}
This paper presents the ageing process of the human body from thehomotopy conceptinthe algebraic topology. The compact connected human body with boundary is assumed to be topologically equivalent to a cylinder. The complexconnected cylindrical shape of the body $x=S^{1} \times I$, described by the functions $f, h: x \rightarrow x$ is a Cartesian product of the vertical interval $I=\lceil 0, \beta\rceil$ and a circle $S^{1}$. The initial state of the body $x=S^{1} \times I$ is the topological shape of the infant. The ageing process called the homotopyisthe family of continuous functions $f_{t}(x)$ on the interval $I=[0, a]$ where $a>\beta$ and $t \geq \beta$. It is an increasing sequence of the function $f_{t}(x)$ of the body $x=S^{1} \times I$. The homotopy relates the topological shape of the infant tothe topological shape of the adult. The homology theory assigns to the human body $(x)$ a sequence of abelian groups $H_{n}(x)$ for $n=0,1,2 \ldots$ and to the continuous function a sequence of homomorphism. The homology group characterizes the number and continuity of the compact surface of human body. The study excludes genetic, hormonal, environmental and other factors in the ageing process and recognizes the fact that ageing continues throughout life, through childhood, and adolescence into adulthood. Topologically the infant is equal to the adult since the infant continuously grows into the adult. The study found an algebraic way of relating homotopy to the process of ageing of human body. It was also established that this could offer other topologists useful topological tips in the application of homotopy to other physical continuous processes.
\end{abstract}

Keywords: Abelian group, ageing, continuity, connectedness, homotopy, homology group, human body, homomorphism, topological space

\section{Introduction}

Obviously, the growth of human body occurs as a result of genetic, hormonal and other environmental factors. But the obviousness of the fact does not stop researchers from making further research works. As the human body experiences changes with respect to time, ageing occurs. Ageing is manifested in all parts of the body since the parts of the body are stuck to one another or have one piece with common surface. The common surface of the body makes it possible to study the behaviour of the body. Theageing of the body is described by continuity and connectivity which are topological properties. The idea of continuity and connectivity have been used in many fields, for instance in the vector calculus. In the vector calculus, it was used to establish the relationship between a line integral around a simple closed curve and a double integral over the plane region bounded by the curve (Massey, 1991). This incredible fact accounts for the fruitful marriage between mathematics and some other disciplines. For instance, in mathematics, specifically algebraic topology, the computable invariant $\alpha(x)=0$ could be used to define the surface a body $x$ that is connected and closed. Thus the surface of a body $x$ is connected and closed if $\alpha(x)=0$. Other existing computable invariants such as Euler characteristics and homology groups havebeen used to study the number of connected components of a space or object (Kinsey, 1993). A common approach is the triangulation of a space or an object. Though, these two algebraic invariants have been used extensively in many fields, their short falls were their inability to clearly distinguish between different connected surfaces of objects or spaces. Several researchers have used homology groups to study digital images (Boxer et al., 2011). With the amount of work already carried out, it was established that theinvariants provided the same information for all the closed and connected surfaces of the spaces or the objects. 
Several attempts are still underway to improve and develop the invariants, thereby making the area an active area of research. A few of the developed invariants that reflect the connectivity properties of spaces or objects werederived from the topological chains (Grandyet al., 2010). Furthermore, the complex spatial structure in the biological systems was also described by topological collections techniques (Giavittoet al., 2003). The Euler characteristics technique could successfully describe the connectivity of the space or objects. All these techniques in addition to other techniques have been employed in many different practical applications (Jafariet al., 2007) but not much has been seen in the direction of the ageing process of the human body.Perhaps, many of the research works have not been focused in this direction. Little recognition has been given to the application of computable invariants to obtain information from the connected spaces or objects in the field of mathematics. In view of this, this paper introduces a topologicalcomputable invariant: the homotopy to describe the process of ageing of human body. Indeed, a research conducted by Issaka et al. $(2016,2017)$ on Fredholm integral equations using homotopy analysis showed how homotopy offers a variety of ways which can be applied to myriad of systems. Of course this study is abstract; therefore, the consideration of the genetic, hormonal, environmental and other factors in the growing process would be discarded. The ageing process is considered by choosing suitable values for the time parameters $t$ of the function $f_{t}(x)$. The values of the parameters $t$ and $x$ in the function $f_{t}(x)$ are adjusted to control the process of the ageing. If for $t \in[0,1]$, there is a homotopy $f_{t}(x)$ from $f(x)$ to $g(x)$ such that $f_{0}(x)=f(x)$ and $f_{1}(x)=g(x)$ then the body is only one year old. Thus, if for $t \in[a, n]$ where $n>a$, there is a continuous function $f_{t}(x)$ called homotopy from one function $f(x)$ to another function $q(x)$ satisfying the condition $f_{a}(x)=f(x)$ and $f_{n}(x)=q(x)$ then the body is described as being $n$ years old. Both the age and the topological shape of the body grow as the homotopy $f_{t}(x)$ increases from $f_{a}(x)=f(x)$ to $f_{n}(x)=g(x)$ for the interval $t \in[a, n]$. It is observed that for the interval $t \in[a, n]$, the supremum $f_{n}(x)=g(x)$ is the actual age of the body. The age increases along 1-dimension. In homology, it is 1-simplex or a line or a path. But the body $x$ is a closed connected surface such that at each change in time variable, the age of the body $x$ increases from one level to another. For instance if $a=f_{0}(x)=f(x) \in x$ and $c=f_{1}(x)=g(x) \in x$ then $a$ and $c$ are connected by a line or path $f_{t}(x)$ given by a continuous map $f:[0,1] \rightarrow x$ in $x$. For this study to be reliable and successful, the study adopted the definitions of continuity and connectivity from various sources.

\section{Main Concept}

\subsection{Basic Definitions and Preliminary}

Definition 1. Let $f: X \rightarrow Y$ be a mapping of one topological space into another. A real function $f(x), x \geq 0$ is said to be continuous at the point $x_{0}$ in $x$ if for each neighbourhood $H$ of $f\left(x_{0}\right)$ there exists a neighbourhood $G$ of $x_{0}$ such that $f(G) \subseteq H \quad$ (Kinsey, 1993).

Definition 2. Let $X \subseteq \mathfrak{R}^{n}$ and $Y \subseteq \mathfrak{R}^{m}$. A function $f: X \rightarrow Y$ is continuous if whenever $y$ is an open set in $Y$, then $f^{-1}(y)$ is an open set in $X$ (Simmons, 1963).

Theorem 1. Let $X \subseteq \mathfrak{R}^{n}$ and $Y \subseteq \mathfrak{R}^{m}$. A function $f: X \rightarrow Y$ is continuous at a point $x \in X$ if and only if for any $\varepsilon>0$, there exists a $\delta>0$, such that whenever $a \in X$ and $\|x-a\|<\delta$, then $\|f(x)-f(a)\|<\varepsilon$ (Simmons, 1963).

In the metric spaces, if $X$ and $Y$ are the two metric spaces with the metrics $d_{1}$ and $d_{2}$ and $f$ is a mapping of $X$ into $Y$ then:

Definition 3. The function $f$ is continuous at the point $x_{0}$ in $X$ if for each $\varepsilon>0$ there exists $\delta>0$ such that $d_{1}\left(x, x_{0}\right)<\delta \Rightarrow d_{2}\left(f(x), f\left(x_{0}\right)\right)<\varepsilon[6]$.

Definition 4. Let $f: X \rightarrow Y$ be topological spaces and $f$ and $g$ continuous functions from $X$ to $Y$. Then $f$ is homotopic to $g$ if there is a continuous family of continuous functions $f_{t}: X \rightarrow Y$ for $0 \leq t \leq 1$ satisfying:

$$
\begin{array}{ll}
\text { i. } & f_{0}=f \\
\text { ii. } & f_{1}=g \\
\text { iii. } & f_{t}(x) \text { is continuous both as a function of } x \in X \text { and as a function of } t \in[0,1] \text { (Kinsey, 1993). }
\end{array}
$$

Definition 5. For any topological space $X$, the abelian group $D$, and integer $n \geq 0$, there is sequence of abelian 
groups $H_{n}(X, D)$ for $n=0,1,2 \ldots$ (Kinsey, 1993).

Lemma 1. If $f$ and $g$ are homotopic, then $H_{n}(f, D)=H_{n}(g, D)$ is the homotopic invariance. It follows that If $X$ and $Y$ are homotopy equivalent, then $H_{n}(X, D)$ is isomorphic to $H_{n}(Y, D)$ (Kinsey, 1993).

Definition 6. A topological space $X$ is connected if it cannot be represented as union $A \cup C$ of two nonempty set open sets $A$ and $C$ which have empty intersection $A \cap C=\phi$ (Simmons, 1963).

Lemma 2. A 1-simplex or a line, a space $X$ is path connected if any two points in $X$ could be connected by 1-simplex or a line. For instance if $a=f(0) \in X$ and $c=f(1) \in X$ then $a$ and $c$ are connected by a line or path $f$ given by a continuous map $f:[0,1] \rightarrow X$ in a topological space $X$ (Kinsey, 1993).

\section{The Homotopy of the Ageing Process}

The study of topology relies on the intrinsic properties of the body. It is coordinate-free since it does not depend on the properties of the chosen coordinates of body. In the study the human body $x$ is consideredin the topological space since our goal is to study the continuous function of the ageing process. The importance of it all is to construct the algebraic invariants such as homotopy that reflect the connectivity of the body. Consider the human body $x=S^{1} \times I$ and let $x \in X$ and $t \in T$ define the growth of the body and the age of the body respectively. Since the final age of the human body is not known let $t=\infty$ represent the final age of the body such that $t \in[\beta, \infty]$ denotes the age interval of the body from $t=\beta$ to $t=\infty$. The time $t=\infty$ is the age threshold value of the human body. The ageing process for all $t \in T$ is the family or the sequence of the functions $f_{t}(x)$ which occurs as $f_{t}(x)$ approaches $g(x)$ for all $g(x) \in \infty$. Suppose $t \in[0, \infty]$ is the interval of the ageing body and $f, g: x \rightarrow x$ are the two functions of the topological shape of the human body,then the shape of the wrinkled body $\infty$ years old is given by the continuous map $f_{t}(x)$ such that $f_{0}(x)=f(x)$ and $f_{\infty}(x)=g(x)$ as shown in Figure 1.

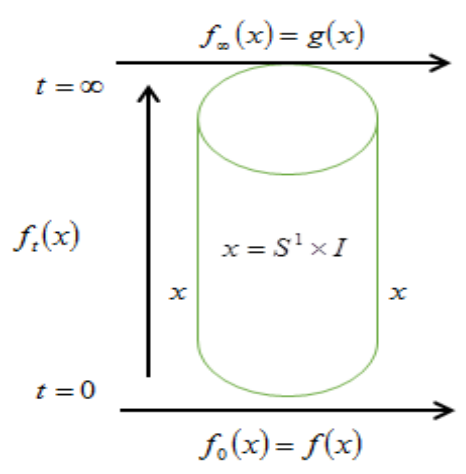

Figure 1. The shape of a wrinkled human body from $f(x)$ to $g(x)$

The closed connected human body $x=S^{1} \times I$ is defined by the functions $f, h: x \rightarrow x$ on the interval $I=[0, \beta]$ where $f$ and $h$ are homotopy equivalences. The interval $I=[0, \beta]$ provides the initial age and height of the whole body $x=S^{1} \times I$. The initial age of the body is $\beta$ years for the time interval $t=[0, \beta]$ since it satisfies the initial condition $f_{0}(x)=f(x)$ and $f_{\beta}(x)=h(x)$. An increase in age occurs as the value $\beta$ increases to the next level. This implies that for an increase in age of $\pi$ from $\beta$, thus $t \in[\beta, \pi]$ there is another function $q(x)$ from $h(x)$ defined by $h, q: x \rightarrow x$ on the interval $t=[\beta, \pi]$ such that the conditions $f_{\beta}(x)=h(x)$ and $f_{\pi}(x)=q(x)$ are satisfied for the homotopy $f_{t}(x)$ from $h(x)$ to $q(x)$ as shown in Figure 2. 


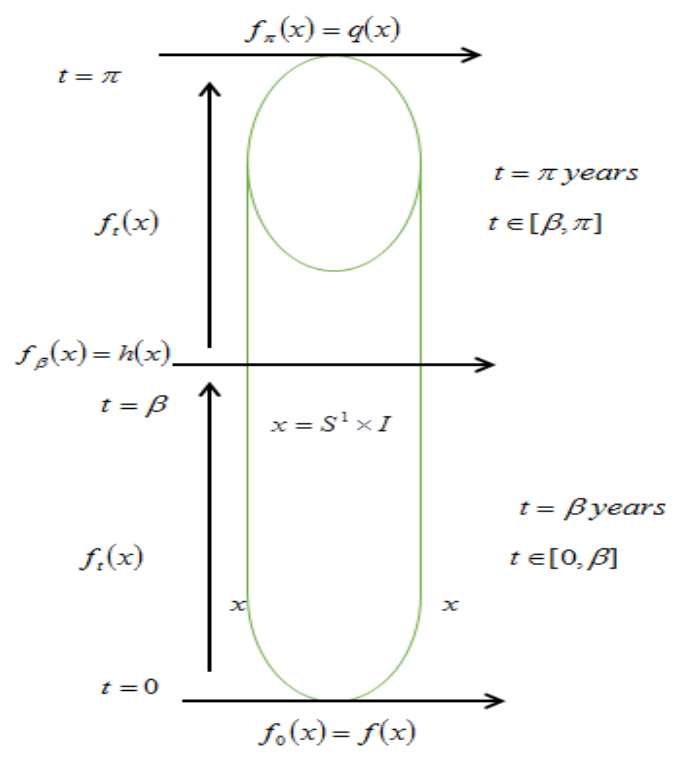

Figure 2. The shape of $(\beta+\pi)$ years body from $f(x)$ to $q(x)$

Figure 2 shows the new age of $(\beta+\pi)$ years of a new body shape. Suppose the functions $f(x), h(x), q(x), k(x)$ and $g(x)$ are continuous functions from $x$ to $x$ such that $f(x)$ is homotopic to $h(x), h(x)$ is homotopic to $q(x)$, $q(x)$ is homotopic to $k(x)$ and $k(x)$ is homotopic to $g(x)$. If there is homotopy $f_{t}(x)$ for $0 \leq t \leq \infty$ from $f(x)$ to $g(x)$ such that $f_{0}(x)=f(x), f_{1}(x)=h(x), f_{2}(x)=q(x), f_{3}(x)=k(x)$ and $f_{\infty}(x)=g(x)$ where the homotopy is continuous both as a function of $x$ and as a function of $t \in[0, \infty]$. Then the body is ageing as illustrated in Figure 1.

Proposition 1. If $f, q: x \rightarrow x$ and $q, h: x \rightarrow x$ are two continuous functions of the body such that $f$ is homotopic to $q(f \sim q)$ and $q$ is homotopic to $h(q \sim h)$ then $q \circ f$ is homotopic to $h \circ q(q \circ f \sim h \circ q)$.

The homotopy $f_{t}(x)$ provides information relating to the age of the body. Generally, for each value of $t>0$, the body experiences increase in both the shape and age when the function $f_{t=0}(x)$ is homotopic to $f_{t>0}(x)$ as illustrated in Figure 2. The topological shapeof each change of the body is the same though; the homotopy $f_{t}(x)$ changes from one level to another for the values of $t \in[0, \infty]$ as shown in Figure 2. For instance in the second year, thus, $0 \leq t \leq 2$, if the homotopy $f_{t}(x)$ is denoted by $f_{1}(x)$ and $f_{2}(x)$, then there is an increase in the age of body from $f_{1}(x)$ to $f_{2}(x)$. $f_{1}(x)$ and $f_{2}(x)$ are the homotopiesfor the functions $f, h, q: x \rightarrow x$ in the interval [0,2]. In a situation where the homotopy $f_{t}(x)$ is identified with the relation $f_{t}(x)=t x f_{0}(x)=0 x=0$ represents the initial ageof the body. As the body grows, thus $t=1,2,3 \ldots, f_{1}(x)=x$ represents the first age of the body, $f_{2}(x)=2 x$ the second age which is twice the first age and $f_{3}(x)=3 x$ the third age of the body. All these continuous functions show the various levels of growths and ages. The increasing sequence of the function $f_{t}(x)$ of the body $x=S^{1} \times I$ provides the chains of the body. We recognized that the initial human body $x=S^{1} \times I$ is a 2-cell complex and that each subsequent growth produces 2-chain represented as $C_{2}(x)$. Thus, each increase in age produces an extra 2-chain complex. Which means the body produces several 2-chains as the age of the body continues to increase. Algebraically, the integer combination of all the 2-chains is denoted by $C_{2}(x)=\lambda$ where $\lambda$ is a positive integer. The 2-cell human body $x$ is an enclosed body hence the kernel of the body which is the set of all the closed compact bodies is $Z_{2}(x)=\lambda$. However, the 2-cell human body is not bounded by any 3-cell complex which implies that the boundary of the 2-cell is zero. Thus $B_{2}(x)=0$. Therefore, the computed 2-dimensional homology group is given as:

$$
H_{2}(x)=\frac{Z_{2}(x)}{B_{2}(x)}=\lambda
$$


Where $H_{2}(x)$ characterizes the connected 2-cell body at each age level. Topologically, the value of the homological invariant $\mathrm{H}_{2}(x)$ indicates that the bodyhas only one closed, connected and compact body but keeps varying as the age increases. The homology theory therefore assigns to the human body $x$ the sequence of abelian groups $H_{2}(x)$ and to the continuous map $f: x \rightarrow x$ a sequence of homomorphisms $f_{2}: H_{2}(x) \rightarrow H_{2}(x)$.

Theorem 2. If $f_{0}, f_{1}: X \rightarrow X$ are homotopic functions, then $f_{0_{2}}=f_{1_{2}}: H_{2}(X) \rightarrow H_{2}(X)$

\section{Conclusion}

The paper proposed the application of the algebraic invariant to a physical phenomenon. The new approach was successfully implemented to establish the growth in the age of the body. Homology group established the correspondence that describes the connected surface of the structure of the body. Homotopyconcepts described the process of ageing of the human body. The age increases as the time variable $t$ increases. The performance of the continuous function $f_{t}(x)$ in the ageing process was significant. The paper provided sufficient evidence to indicate that the homotopy approach is reliable to the study of the process of ageing of human body. The most important contribution of this paper is the description of the process of ageing within the framework of algebraic topology.

\section{References}

Boxer, L., Karaca, I., \& Oztel, A. (2011). Topological invariants in “ Digital Images, Journal of Mathematical Sciences: Advances and Application, 11(2), 109-140.

Giavitto, J. L., \& Michel, O. (2003). Modelling the Topological Organization of Cellular processes, Biosystems, 70(2), 149-163. https://doi.org/10.1016/S0303-2647(03)00037-6

Grandy, L., \& Polimeni, J. (2010). Discrete Calculus: Applied Analysis of Graphics for Computational Science, Springer, New York.

Issaka, I., Obeng-Denteh, W., Mensah, P. A. A., \& Owusu-Mensah, I. (2016). Homotopy Analysis Method for Solving Fredholm Integral Equations of the Second Kind. Bulletin of Mathematics and Statistics Research, 4(1), 167- 173. https://doi.org/10.9734/ARJOM/2017/32585

Issaka, I., Obeng-Denteh, W., Owusu-Mensah, I., Prempeh, E., \& Mensah, P. A. A. (2017). On the Regularization-Homotopy Analysis Method for Linear and Nonlinear Fredholm Integral Equations of the First Kind, Asian Research Journal of Mathematics, 4(1), 1- 13. https://doi.org/10.9734/ARJOM/2017/32585

Jafari, H., \& Momani, S. (2007). Solving fractional diffusion and wave equations by modified homotopy perturbation method, Phys, Lett, A(370), 388-96. https://doi.org/10.1016/j.physleta.2007.05.118

Kinsey, L. (1993). Topology of surfaces, Springer-erlag, New York, Inc. USA, 279. https://doi.org/10.1007/978-1-4612-0899-0

Massey, W. S. (1991). A Basic Course in Algebraic Topology, Springer-erlag, New York, Inc. USA, 428. https://doi.org/10.1007/978-1-4939-9063-4

Simmons, G. F. (1963). Introduction to Topology and Modern Analysis, McGraw-Hill book Company, Inc. New York, USA, 362.

\section{Copyrights}

Copyright for this articleis retained by the author(s), with first publication rights granted to the journal.

This is an open-access article distributed under the terms and conditions of the CreativeCommons Attribution license (http://creativecommons.org/licenses/by/4.0/). 